\title{
Three-course graduate electro-optics laboratory sequence at the University of Dayton
}

Perry Yaney, Bradley Duncan, Gordon Little

Perry P. Yaney, Bradley D. Duncan, Gordon R. Little, "Three-course graduate electro-optics laboratory sequence at the University of Dayton," Proc. SPIE 4588, Seventh International Conference on Education and Training in Optics and Photonics, (28 May 2002); doi: 10.1117/12.468713

SPIE Event: Education and Training in Optics and Photonics 2001, 2001, Singapore, Singapore 


\title{
Three-course graduate electro-optics laboratory sequence at the University of Dayton
}

\author{
Perry Yaney ${ }^{*}$, Brad Duncan ${ }^{* * b}$, Gordon Little ${ }^{* * * c}$ \\ ${ }^{\mathrm{a}}$ Department of Physics, ${ }^{\mathrm{b}}$ Department of Electrical and Computing Engineering, ${ }^{\mathrm{c}}$ Research Institute, \\ University of Dayton
}

\begin{abstract}
The UD Electro-Optics Graduate Program offers the M.S. and Ph.D. degrees. It is an interdisciplinary program between the Electrical and Computer Engineering Department and the Physics Department and is designed for students with a B.S. in either of these fields. In order to strengthen skills in applied optics, optical measurement techniques, photonics and data acquisition and analysis methods, a required three-course laboratory sequence was designed. The first course in the fall term has seven basic optics projects that include focal length measurements, lens systems, radiation detection, polarization, interference, near and far field diffraction, interferometry and coherence. The first half of the second course, given in the summer term, covers fiber optics and fiber optical systems. In the second half, the students propose, design, construct, test and report on an electro-optical/photonics system. The third course in the fall term has five advanced projects on the topics of optical spectroscopy, holography, characterization of lasers, laser Doppler velocimetry and optical pattern recognition. Details on the design of these courses are presented along with examples of student work and the results of student evaluations and responses to the lab program.
\end{abstract}

Keywords: Optics education, laboratory instruction, optics, electro-optics, photonics

\section{INTRODUCTION}

Around 1980, several members of the Physics Department of the College of Arts and Sciences, the Electrical Engineering Department of the School of Engineering and the University of Dayton Research Institute (UDRI) started serious conversations about forming an interdisciplinary academic program based on the optics strengths of these two academic departments and UDRI. This idea had been discussed for several years. These strengths included more than a dozen Ph.D. faculty and researchers experienced in a wide range of optics and optics-related fields plus several million dollars of funding in these fields. In 1981, the University of Dayton (UD) announced an Advanced Technology Challenge Grant initiative and request for proposals to stimulate the development of a new technology graduate program with an award of $\$ 300,000$ startup funds. A committee of nine from the above organizations was formed to prepare the proposal. This committee set down the following goals for the proposed program: 1) The program should be an applied science and engineering program based in the School of Engineering. 2) The proposed program was entitled Electro-Optics (EO) to emphasize the joining of the Electrical Engineering and Physics academic departments with UDRI into an interdisciplinary graduate program. 3) Although the first phase of the program was to offer the Masters Degree, the program must lead eventually to the granting of the Ph.D. degree; 4) Except for certain rare cases, a thesis would be required of all Masters degree candidates. 5) In order that the graduates from this program have a reasonable exposure to hands-on optics, optical devices and systems, photonics, electronic data acquisition, and data processing and analysis, a supervised, three-laboratory course sequence was proposed to be an integral part of the core program. The proposal for an Electro-Optics Graduate Program won the award in 1983. The last goal given above is the subject of this paper.

The EO Program has granted 151 M.S. degrees since 1984 and 16 Ph.D. degrees since the inclusion of the Ph.D. degree in 1994. All graduates have gone through the three-lab sequence. Almost without exception, these graduates have responded positively to this lab program. The most common complaint is that the large amount of time required to complete the

\footnotetext{
*Perry.Yaney@notes.udayton.edu; phone 937-229-2221; fax 937-229-2180; http://www.udayton.edu; University of Dayton, 300 College Park, Dayton, OH 45429-2314; ***Bad.Duncan@notes.udayton.edu; phone 937-229-2796; fax 937-229-2097; Electro-Optics Graduate Program, 300 College Park, University of Dayton, Dayton, OH 45429-0245; ${ }^{* * *}$ glittle@ spectra-research.com; phone 937299-5999; fax 937-299-7773; Spectra Research, Inc., 3085 Woodman Dr., Dayton, OH 45420-1173
} 
projects does not seem reasonable in terms of the one-credit-hour allotted for each lab course. The response usually given to this complaint is that any lab course provides a student with the opportunity to develop their eye-brain-hands skills in applying the knowledge gained in the classroom and book studies to designing,, building and making things work in the real world. The development of these skills is a personal matter different for every student that involves very little new academic material and, as such, does not warrant additional academic credit. A supervised academic laboratory course is a unique and safe environment for students to explore and sharpen their skills and competency in their chosen field of study. Typically, the gain in value that students experience from a supervised lab course is related to the investment of effort and time they dedicate to the course in much the same way as compound interest works. These labs are mildly structured to ensure that the efforts of the students have the greatest opportunity for successful experiences and learning. By supervising these labs, we can effectively challenge the students to develop their self-learning and error-correcting skills.

From the teaching viewpoint, offering three graduate labs requires considerable commitment and organization. Each course is made up of a set of projects that are assigned to all students. These projects have specified goals that give the students exposure to a wide range of experiences in many optics areas including optoelectronics and photonics as well as to optical techniques, electronic instrumentation, data acquisition schemes and data analysis techniques. Each project allows the student to see how the appropriate theory is applied to a real situation and an opportunity to compare measurements and observations to calculations based on theoretical models. Within each lab course, the students are typically rotated among the projects in a scheduled manner based on the number of students. Since teamwork is an important part of the lab program, student groups typically of two or four students each are arranged at the beginning of each course.

The EO Program Graduate Laboratory facility consists of six lab rooms and a supply/computer room all connected by a hallway. Three of the lab rooms have $4 \mathrm{ft}$. $\mathrm{x} 8 \mathrm{ft}$ optics tables for projects requiring large areas and the remaining three labs have $3 \mathrm{ft}$ x $4 \mathrm{ft}$ optics tables. All labs have workbenches with drawers and cabinets. Each of the labs can be closed off to achieve a reasonable degree of darkness. Typically, in each of the three lab courses, there are four to six different projects running simultaneously. Since the lab courses are staggered over the calendar year so that only one course is offered in any given term, this arrangement makes only modest equipment demands for maintaining the lab program. Moreover, since each course has a lab procedure manual, the short-term burden on the instructor of each course is focused on getting the students started on each project and providing guidance when a particular group has difficulties. The long-term burden is equipment repair and replacement, updating projects and the manual, computer maintenance and software upgrade.

The three-lab sequence consists of the Geometrical and Physical Optics Laboratory, offered in January, the Electro-Optics Systems Laboratory, offered in May, and the Advanced Electro-Optics Laboratory, offered in August. For a student matriculating in August, the lab sequence starts in the second term (January) of the student's first academic year. This arrangement provides the students with the essential first-term courses of Geometrical Optics, Optical radiation and Matter, and Linear Systems and Fourier Optics before starting the lab sequence. Concurrent with the first lab course, the student takes Introduction to Lasers, Electro-Optical Devices and Systems, and Guided Wave Optics. The second lab course, offered in the summer term, consists of fiber optics projects during the first half of the term and independent group projects during the last half. The third lab course, offered in the fall, is a set of five two-and-a-half-week, open-ended projects that give the students experience in learning how to apply their knowledge and the experience gained in the previous two lab courses to build up a project from scratch that is new to them and to achieve meaningful results. Throughout these lab courses, teamwork is emphasized not only between the members of within each group, but also between the groups. In the third lab, it is a general principle that a group who has already done a particular project is the first resource for a group that is just starting that project. Moreover, the students are encouraged to share their data in instances where a particular group was unable to acquire good data for a particular project, of course, with stated credit to the sharing group. The lab courses have a variety of types of assigned tasks ranging from formal written reports to detailed theoretical calculations and computer modeling.

Other issues such as neatness, responsibility for equipment, sharing of the work load, ethical behavior (honor code), polite demeanor and regular attendance are discussed and expected of all students. The following sections describe in some detail the content of each of the three laboratory courses with some examples of student work. The final section gives the results of a survey of EO graduates and an assessment of the three-lab sequence.

\section{THREE-LAB SEQUENCE}

\subsection{Geometrical and Physical Optics Laboratory}

During this course, each student performs seven projects. These projects were chosen to explore topics covered in the first- 
term lecture courses of the core program and to develop the basic skills required for designing, setting up and carrying out optical projects. The projects are as follows:

1) Determination of the focal lengths of two lenses by six methods

2) Characterization of simple lens systems - the beam expander and the relay lens system

3) The Airy disc and Fraunhofer diffraction - the diffraction limit, single and double slits and spatial filtering

4) Detection of radiation - silicon photodiodes and lock-in detection

5) Fresnel diffraction by a opaque, narrow obstacle and a slit

6) Polarized light - polarizers, birefringent materials, wave plates and Brewster's angle

7) Interferometry and source coherence measurements using a Michelson interferometer

Before undertaking each project, each student receives a description of the problem. Each project requires two class sessions to complete (one scheduled session per week) and a report describing the work is submitted one week after the second session. To make optimum use of laboratory time, students are expected to read over the project description and to organize their thoughts concerning the required procedures and background before the beginning of the laboratory session.

Each student is expected to keep a lab notebook and all pertinent information concerning the projects is to be recorded in this notebook. This includes a list of the equipment used, the procedure followed, the primary data taken, calculations and results, pertinent observations and interpretation of data.

The reports on which the course grade depends consist of a maximum of five pages of text plus graphs, tables, diagrams, etc. The outline of the report includes:

1) Short introduction to the problem

2) Brief discussion of the theory

3) Description of the procedure used

4) Presentation and analysis of results

5) Conclusions

\subsection{Electro-Optics Systems Laboratory}

The intent of this course is to provide the student with the opportunity to explore the basic elements of fiber optics and experimental optical systems and technology. To this end, the course is divided into two major activities of concentrated study. In the first half of the summer, student groups perform the various projects outlined in the "Projects in Fiber Optics Applications Handbook" provided with the Newport Fiber Optic equipment kits. ${ }^{1}$ In the second half, student groups perform a demonstration level experimental "mini-project" of their choosing. This consists of independent project-oriented investigations of electro/fiber-optic/optoelectronic systems and devices in general, which include sources, detectors, image processing, sensor instrumentation and integration, electro-optic components, display technology, and nonlinear optical devices and systems.

Each student maintains a lab notebook that documents the student's efforts in carrying out the projects and the results obtained from the fiber optic kit projects as well as providing evidence of the student's individual participation and efforts in the group mini-project. Additionally, the students prepare pre-lab write-ups before attempting any fiber optic kit project and before beginning the group mini-project. These graded write-ups identify the essential goals, procedures and equipment necessary to perform a given project and include any required preliminary calculations.

\subsubsection{Fiber Optics Projects}

The Newport fiber optic kit has following projects:

1) Handling Fibers, numerical aperture

2) Fiber attenuation

3) Single-mode fibers I

4) Single-mode fibers II

5) Coupling fibers to semiconductor sources

6) Connectors and splices

7) Components for fiber communication

8) Fiber optic communication link

9) Multimode intensity sources

10) Single-mode interferometric sensors 
Each group has access to one of the three fiber optics project kits for six weeks. Each kit contains a different set of projects. At midterm (about six weeks into the term), primary access to each kit is exchanged among the groups to insure that all groups have appropriate opportunity to do all projects. All of the above projects, with the exception of project 6 , are carried out and recorded in the laboratory notebook.

\subsubsection{Experimental mini-project}

The instructor assists the students in identifying acceptable projects, if necessary. The mini-project may investigate any facet of electro-optical engineering or the optical sciences of interest, yet should generally be designed so as to demonstrate either results presented in a recently published technical journal article or textbook principles not demonstrated as part of a previous laboratory course. For this course, the students divide themselves into groups of two to four students each. Each group is required to submit for grading a proposal and summary of their mini-project for which the students are jointly and severally responsible for the preparation. The students in each group receive the same grade for the mini-project. All mini-projects are performed on the University of Dayton campus using University equipment. At the end of the summer, each group is required to demonstrate their mini-project to the instructor and the class. This demonstration is not formal or elaborate, but is intended to communicate the project and show the results. Visual aids are encouraged. In addition, a mini-project summary, not to exceed ten pages, is also due at this time.

\subsection{Advanced Electro-Optics Laboratory}

\subsubsection{Course design and goals}

This lab course consists of five projects that each group carries out over two and a half weeks in the fall term. These projects require that the students study a variety of topics and develop skills for working with a range of optical elements, electronic equipment, lasers, methods of data acquisition and data analysis schemes. This course is intended to provide the students with learning experiences that utilize much of the academic material covered since starting the EO Program. It is hoped that they will bring the abstract knowledge gathered from the lecture courses into real-world observations and measurements. Technique and skill development are necessary parts of this process that, although guided by the instructor, are the student's responsibility. The learning curves of the students vary widely and are time-consuming, which results in students needing considerably more time to carry out a given project than one or two afternoons, which students soon learn. The instructor meets formally with the lab class for an afternoon once each week and is available for consultation during other times to the extent possible. For the first 30 minutes or so, each group presents their current results to the class. This provides opportunity for discussion and instructor input. Efficient and generous communication between students and between instructor and students help to minimize the time expenditure on any particular project. Teamwork, sharing and openness is encouraged in order to enhance free exchange of experience, knowledge and questions among the students.

The primary guidelines for designing this lab course were the following: 1) Lab projects should be reasonably self-contained and somewhat open-ended with some well-defined measurement goals. 2) Projects should provide the students with a variety of areas to explore. 3) Each group should have the opportunity to successfully use an optical-electronic system that was set up by a previous group. 4) Students cannot possibly be exposed to every facet of the fields of optics, electro-optics and optoelectronics in this lab course. 5) The projects should give students the experience and confidence that they can come up to speed on any electro-optic area regardless of whether or not they have worked directly with that area in the EO Program. 6) The students should learn how to absorb not only knowledge and technique from reference materials, but also from coworkers. 7) The subject matter of the projects should relate to real, applied situations that occur in industrial, commercial and/or governmental organizations. These guidelines can be satisfied by a wide variety of project choices. The projects described below represent one set of choices that meet the above guidelines even though they were set up at the beginning of the EO Program in 1983. This issue is further discussed in the Conclusions Section.

A lab manual and the accompanying handouts provide procedural guidelines that range from a few paragraphs to step-by-step descriptions. It was intended that the projects be somewhat open-ended. In other words, the students must fill in some details that are not provided by the manual. They do this by talking to other students or the instructor. Discussing techniques, trouble-shooting and following verbal instructions are the skills developed here. These projects are not "cookbook" in that there are many details and procedures which require background reading and development by the student. This reading and development for all projects is each student's responsibility the evidence of which shows up in the lab notebooks of the student groups.

Each group records all pertinent information, plots, photos, computer programs and data, etc. for all projects in a three-ring lab notebook, which is graded at the end of the term. All textual and tabular material is to be written either in ink or with a 
word processor (preferred). Each student is expected to select a topic for a paper study for which a formal proposal is written. This topic is selected in consultation with the instructor and no two students in the lab class can chose the same topic. The topics generally are in some way related to the projects in the lab course. There is no experimental work done for these studies. Following the return of the graded proposal, the students write formal reports on their topics, which are graded. The lengths and formats of the proposal and the report are given in some detail. The goal of this activity is to expose students to proposal and report writing on topics that are somewhat new to them. At the end of the term, each student gives a 10 minute formal presentation (viewgraph or computer) on their topic to the class and instructor.

\subsubsection{Project goals and tasks}

\section{Optical spectroscopy:}

1) Learn to use the processor-controlled grating spectrometer.

b) Learn to use the A/D computer data acquisition system and software.

2) Learn to use the photomultiplier detection system and the data acquisition system with the scanning spectrometer to obtain undistorted spectra.

3) Record and analyze the emission spectrum of a low pressure Hg lamp and identify the buffer gas.

4) Record and analyze the lasing and spontaneous emission spectra from a He-Ne laser.

5) Record and analyze the emission spectra from a diode laser below, at and above threshold.

6) Record the absorption spectrum of a crystalline laser material. Calibrate, do background normalization and determine the integrated cross sections of the recorded groups of absorption lines. Compare to published data.

\section{Holographic techniques:}

1) Review the theory of holography and learn how to make holograms. Determine exposure times using a calibrated power meter and the given sensitivity of the recording medium.

2) Record and demonstrate a single-beam hologram.

3) Record and demonstrate a two-beam (side band) hologram.

4) Record and demonstrate a reflection hologram.

5) Record and demonstrate double-exposure and real-time interferometric holograms.

6) Examine the influence of spatial and temporal coherence on the formation of the holographic image.

\section{Application of interferometry - LDV:}

1) Review laser Doppler methods of measuring velocity.

2) Align the optics to obtain suitable beat frequency bursts on a digital storage oscilloscope using photomultiplier detection.

3) Measure the axial velocity profile of laminar water flow in a glass tube using laser Doppler velocimetry for two flow rates.

4) Analyze the data and compare the observed profiles with the predicted profiles based on laminar pipe flow theory.

\section{Laser characterization:}

He-Ne laser:

1) Review the characteristics of a confocal cavity as applied to an optical spectrum analyzer

2) Computer record the mode spectrum and the mode drift using the spectrum analyzer and a digital storage oscilloscope with an IEEE-488 interface.

3) Analyze the spacing and width of the mode data and compare to expected values. Deduce and calculate the source of the mode drift.

Argon ion laser:

1) Become familiar with the argon ion laser.

2) Measure the power performance of an argon ion laser and determine the slope efficiency, the "wall-plug" efficiency and the threshold for lasing.

3) Computer record the beam profiles of an argon ion laser at four cavity lengths from the laser using a pyroelectric linear array detector with the digital storage oscilloscope.

4) Determine the $\mathrm{e}^{-2}$ widths of the beam profiles by fitting Gaussian functions to the recorded profiles. 
5) Fit the resonate cavity equation for the argon ion laser to the profile widths and compute the curvature of the output coupler of the laser.

Pulsed/Q-switched Nd:YAG laser:

1) Measure the energy performance and determine the slope efficiency, the "wall-plug" efficiency and the threshold for lasing.

2) Measure the Q-switched pulse width versus input pulse energy and compare to theory

3) Record the non-Q-switched relaxation oscillations and compare to theory

4) Record and analyze the mode beating in the pulse using a high-speed digital storage oscilloscope

5) Review second harmonic conversion.

6) Measure the efficiency of second harmonic conversion using a Q-switched Nd:YAG laser.

\section{Optical pattern recognition:}

1) Review optical pattern recognition

2) Align a frequency-plane-correlator/matched-spatial-filter system and the determine the conditions for recording a matched filter

3) Produce a Vander Lugt matched filter.

4) Set up and demonstrate the operation of the frequency plane correlator system using a CCD camera. Record both correlation and convolution images.

5) Characterize the performance of the correlator system using a frame grabber and image processing. Determine the threshold for correlation.

\section{EXAMPLES OF STUDENT WORK}

Each of the three lab courses provides the students with a variety of opportunities to make precise measurements on some feature of or with an electro-optical system. The emphasis is generally to match up the theory to the measurements either by direct calculation from first principles or by nonlinear fit of the theoretical expression to the data. These measurement and analysis tasks are intended to help the student see how theory is used to characterize a series of measurements made on or with an optical system. Figure 1 shows the results of the calculation and measurement of the Fresnel diffraction pattern produced by a long, thin obstacle of about $1.0 \mathrm{~mm}$ in diameter. In this case, the objective is to show how theory directly predicts with reasonable accuracy the observed pattern. It also, shows how the measurements may not reveal all the detail given by the theory. This realization vividly brings out the issue of how the measurements were made and points to the fact that to achieve good

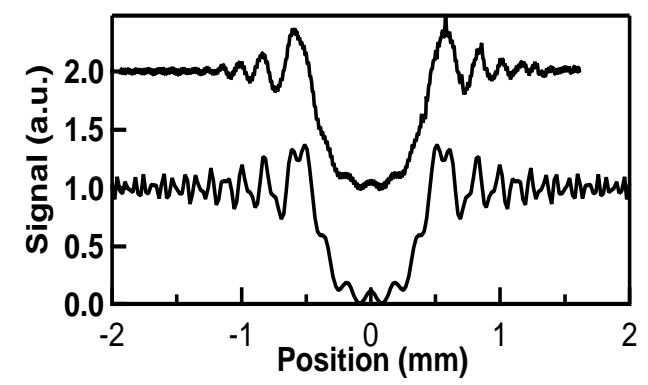

Fig. 1: Diffraction around an obstacle. Upper plot is measured and lower plot is theory. agreement with theory requires attention to detail. Clearly the resolution of the scan in Fig. 1 was not adequate to show the detail in the pattern in the measured data. However, it is also evident that the student was coping with a noise problem, which would have been aggravated by narrowing the scanning aperture. Thus, the student had to deal with the classic problem of signal-to-noise ratio versus resolution and scan time.

Figure 2 is the screen of the digital storage oscilloscope (DSO) obtained with the linear array detector on which the beam of an argon ion laser is incident. This screen was uploaded to a computer via the IEEE-488 interface. The student is challenged to extract the data from Fig.2. Students have done this simply by measuring the height of each signal step with a ruler on a printout, by using the cursor functions in a graphing program ${ }^{2}$ or by writing a program to extract the heights. Typical results of the data reduction, curve fitting and graphing are shown in Fig. 3. (Students are encouraged to use Igor Pro, which is a powerful graphing and analysis software program. ${ }^{2}$ ) In this project, students use curve fitting to extract the $1 / \mathrm{e}^{2}$ width of the beam profiles. This provides beam radius versus the distance from the back mirror of the laser mirror cavity. This mirror is flat and, therefore, marks the location of the waist of the beam. Typically, the students record and analyze the beam profiles at four distances to obtain the beam expansion function. Since the spacing of the cavity mirrors is given, then the Gaussian beam expansion function for this cavity can be fit to the observed function from which the curvature of output coupler is a fitting parameter. This activity is intended to help the students see the characteristics of Gaussian beams by direct measurement and to give them experience in modeling optical performance using nonlinear regression analysis. 


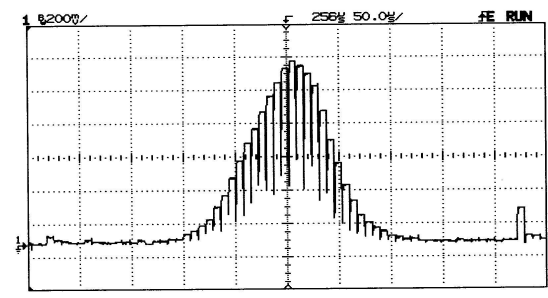

Fig. 2: Computer-recorded digital storage oscilloscope display of an argon ion beam profile.

The final example of student work is the application the Doppler effect and heterodyning to measuring the velocity of fluid flow. In this particular instance, water flow in a glass tube with a $1-\mathrm{cm}$ inner diameter is measured. Uniform pipe flow is established using a $75-\mathrm{cm}$ long tube. The volume flow rate is set to insure laminar flow, which results in the classic parabolic transverse velocity profile. In this project, two beams split from the same laser beam cross inside the tube. The light scattered from naturally occurring particles moving through the crossing volume is collected via a lens, focused onto a pinhole and detected by a photomultplier. The two beams interfere to produce a fringe field in the crossing volume. Passage of a particle through this fringe field ultimately produces a beat frequency within the scattered light profile of the particle, which is recorded on a DSO. The students must record sufficient data to obtain statistically valid average velocities across the diameter of the flow tube. The measured velocities are shown in Fig. 4 as well as the parabolic fit to the data. The students are given reference material that allows them to compare their fit to theory and to the volume flow rate measured with a calibrated ball-in-glass flowmeter. This project provides an example of an application of optical techniques to a practical measurement scenario that is often used in aeronautics and other fields where remote measurements of gas or fluid flow velocity are needed. The alignment of this optical system can be rather tedious, which often inspires the students to try to devise ways of accomplishing the alignment. One of the points to be learned in doing this project is that to make an optical technique work often requires thoughtful design and careful alignment with attention paid to details, such as polarization, relative amplitude of the beams, focus, spatial filtering and triggering level of the DSO.

\section{SURVEY AND CONCLUSIONS}

\subsection{Survey}

Since the beginning of the UD EO Program, students have responded positively to the three-lab sequence when asked for their opinion and, of course, the usual course evaluations were mostly positive. Probably the most negative comments typically concern the amount of time that the labs required in comparison to the one credit hour assigned to the labs. Usually, when the student understands that credit hours are not intended to have a linear relationship with the amount of work required for lab work as discussed in the Introduction, they accept what the lab program is providing for them in a positive way.

In order to document the reactions and opinions regarding the three-lab program, we recently sent out a simple survey by email to the EO graduates. Although we had email addresses for only about one sixth of our graduates (27), all of the surveys were returned. In addition, we asked for comments about the three-lab program. The survey was the following: 
Please respond with a number of $\mathbf{1}$ to $\mathbf{5}$ according to the following key:

1. Strongly agree; 2 . Agree; 3 . Agree with reservations; 4. Disagree; 5 . Strongly disagree

The UD EO three-lab sequence

(a) is a desirable feature of the UD EO Program

(b) challenged you in a meaningful way

(c) helped you to understand better the material covered in the lecture courses

(d) increased your confidence in finding and performing in your first EO job

(e) should be reduced to one or two structured labs

(f) should be replaced by an unsupervised, open lab

(g) should be discontinued and replaced by lecture courses

The results of the survey of the EO graduates are displayed in Figure 5. Responses were given to all statements except for four respondents who are working on their doctorates and responded "not applicable" to (d). The responses to the survey include EO graduates from every year since the program began except for 1995 and 1997 (the first graduate was in December 1984). Ninety-six percent of the responses strongly confirmed that the three-lab program is desirable in statement (a) and firmly rejected the proposal to discontinue the lab program and replace the labs with lecture courses in statement $(\mathrm{g})$. A similar survey was sent out to our current students, but the number of responses was small; however, the pattern was quite similar. These results are consistent with the stories from our graduates who describe how quickly they were able to become productive in their first job. Some of the comments sent in with the survey responses from graduates are as follows:

"I really think the labs were a major part of the EO program and were a big benefit to me in the coursework and after I took a job."

"I can't say enough about the UD EO lab program.......... The UD EO lab series confirmed, affirmed and further developed my intense love for laboratory research activity."
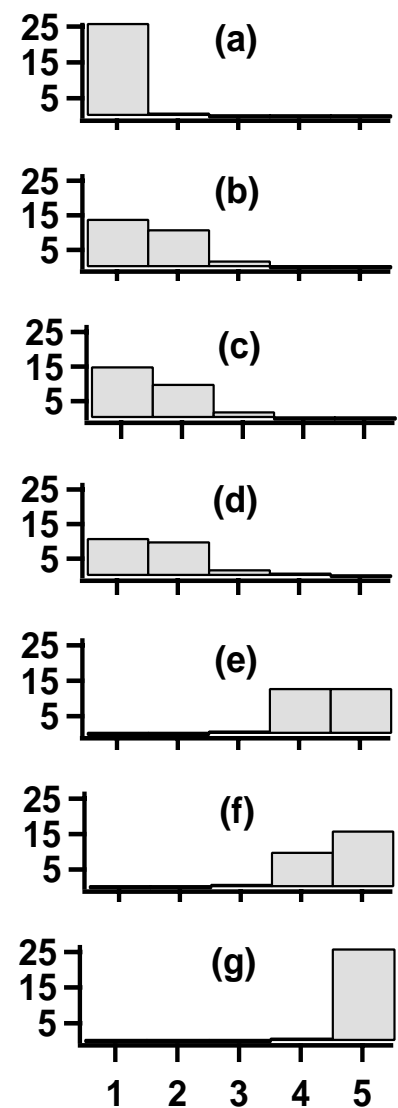

Fig. 5: Survey of UD EO graduates (see text for key).

"I work in an optics lab on a daily basis. The skills that I acquired in the EO labs are used routinely, and it surprises me that some individuals with whom I have worked do not have these skills. Extensive lab training in an academic setting gives you the confidence to solve problems when real problems are encountered on the job."

"The lab program was an important learning tool for myself and others when I went through the program and I think it should still be important. It provides pertinent, timely, and insightful experiences for the participants. If a picture tells a thousand words, then an experiment gives the student an opportunity to 'paint' a picture with learned theories."

"I felt well prepared by the EO program and the 'hands-on' experience of both the laboratory courses and the research assistantship I held in the mid 1980s. As an experimental physicist in an applied engineering field, UD played an important role in shaping my career path."

"I have greatly benefited from UD's EO lab program and hope that it will continue to grow. In the past five years, I have worked for two companies in which "hands-on" experience was a definite benefit. Both positions required that I draw on my lab experience in optical measurements and testing......... I believe that lab experience gives the student an opportunity to hone their research and analytical skills, giving them an edge over those who have had very little "hands-on" laboratory experience."

"Specially for those of us, who had their master's thesis topic rather theoretical/computational in nature, the labs gave us the confidence that we could survive if we were to do experimental optics." 
"Lab exercises are extremely important for optical engineers. The hands-on labs help solidify what is taught in class and they build confidence in working with equipment."

"It is the first exposure most of us have to actually touch optics, tweak optics, even break some optics. Without the pressure of having to perform for a job, we can experiment, teach ourselves how things work in the lab, and how to experiment. Overall, I would call the labs a necessity not an option and really it is what sets UD apart from the other programs at that level."

\subsection{Conclusions}

There are several key stages in designing a structured laboratory sequence such as in the UD EO lab program. 1) There must be a clear vision of the goal of the lab program. 2) This vision and the goal should provide the conceptual framework from which the projects and tasks in each lab course can be designed. 3) These project and task designs provide the basis for determining the equipment, component and supply needs of the lab courses. This step requires a solid familiarity with the vendors who sell the types of equipment, components and supplies needed. 4) The project and task designs provide the basis for determining the space needs. The UD EO lab program was initially designed around six $4 \mathrm{ft} \times 8 \mathrm{ft}$ optics tables. Unfortunately, the space originally available was cut by about 30\%. This required some creative redesign wherein we replaced three of the tables with smaller $3 \mathrm{ft} \times 4 \mathrm{ft}$ optics tables. These tables have turned out to be quite acceptable. The smaller projects are set up these tables and it has become apparent that the smaller size provides the students with experience in making optical setups compact. 5) Besides the optics tables, the furniture in the space must provide adequate and convenient storage plus bench top and shelf space for mounting electronic equipment, recording data, etc. 6) The design of the space must include methods of darkening individual lab areas without affecting other labs as well as access and egress routes for each optics lab area. 7) Of course, the design must include all the necessary electrical power, lighting, cooling water, sinks, etc. 8) Important needs for modern electro-optics laboratories are computers and data acquisition systems. These systems have significant space needs and usually need to be portable. 9) Because of the wide variety of projects, typically a team of two or three experienced individuals is needed to select the equipment, components and supplies for each lab and then to bring each lab to operational status. 10) Perhaps the most tedious and time consuming requirements are ordering the equipment, components and supplies, organizing them in the lab areas and keeping them that way, and then maintaining and replacing them as needed. 11) The final steps have to do with writing procedures or a lab manual for each lab course, developing the projects so that they can be accomplished in reasonable periods of time, updating the manual as needed and finally identifying activities and projects that should by either updated or replaced. The final demand that this kind of lab program makes is for an instructor to learn the projects in a given lab well enough to guide the students and trouble shoot the projects when problems arise.

The UD EO lab program has successfully moved through all the above stages. The results cited in the previous section give solid evidence to this claim. Our experience shows that it is not necessary and it is probably impossible to touch on every optical, electro-optical, photonic and electronic topic in a lab sequence design. The important feature of the type of supervised lab program described here is that it provides students with well-defined areas for investigation that have not only clearly defined goals, but also provides opportunities for exploration. The espoused principle is that it is the process of learning to do lab work and all that it entails within a supervised and appropriately structured program that is the key to opening the door to the worlds of experimental science and hands-on engineering to students. The role of the instructor in this design is both that of a mentor and that of a teacher. One of the many lessons students learn is that no matter how evident the theory is regarding a given project, making an optical/electro-optical/optoelectronic/photonic system work is another matter that cannot be learned from a book, but just has to be experienced. They personally experience that things do not always work right, namely, equipment fails, optics are not perfect and the alignment may be wrong. More importantly, they learn to recognize these situations and how to correct or avoid them. One of the most important skills that we observe in students progressing through our lab sequence is the growth of their ability to critique their own work and to correct their own errors. Thus, despite the demand on the authors as well as on the students since the beginning of the EO Program, our experience with a supervised and structured graduate laboratory sequence has been very positive and productive for our graduates. The following quotation from an EO graduate sums it up succinctly: "I agree with having structured labs. I think solid thesis work should cover "unstructured' lab work."

\section{ACKNOWLEDGMENTS}

The authors thank the University of Dayton for awarding the Advanced Technology Challenge Grant to the Electro-Optics Program proposal in 1983 and the anonymous donor who provided the matching funds that made the grant possible. We want to remember and thank the members of the EO proposal committee for their years of thinking, conversing and then writing the proposal for a new 
interdisciplinary graduate program. All of these individuals fully embraced the design of the EO Program as outlined in the Introduction. The members of that committee were James Harris (UDRI, Optical Engineering Group; current location unknown), Lloyd Huff (Applied Physics Division, UDRI; retired), Donald Lewis (EE; deceased), John Loomis (Optical Engineering Group, UDRI; currently EE), J. Michael O'Hare (Physics chair and the lead person in writing the Ph.D. proposal), James Schneider (Physics; deceased), Gary Thiele (EE; first EO Program Director), Tommy Williamson (EE; retired) and Perry Yaney (Physics). One of us (PY) wants to recognize his colleague and good friend James Schneider for his dedication to the idea of an EO program and for his help in designing of the EO Graduate Laboratory facility in the Kettering Engineering and Research Laboratory Building. Thanks goes to Robert Hengehold (Chair, Engineering Physics Dept.) of the Air Force Institute of Technology for his suggestions regarding projects for the first lab course of the sequence. We wish to thank the many individuals at UD who have supported this program in varied ways, especially Gene Gerber (retired), who headed up the UDRI Optical Engineering Group during the 1980's, and his staff. Their regular support of many EO students during the early years greatly aided the Program in achieving the stability and success it now enjoys. Finally, we want acknowledge the School of Engineering and the College of Arts and Sciences for their continued support of the fully interdisciplinary EO Graduate Program.

\section{REFERENCES}

1. Newport Corporation, 1791 Deere Ave., Irvine, CA 92606; www.newport.com

2. Igor Pro version 4, Wavemetrics, Inc., P.O. Box 2088, Lake Oswego, OR 97035; www.wavemetrics.com 